Tatsuro Kondoh • Tadashi Matsumoto • Makoto Ochi Kazuko Sukegawa • Yoshiro Tsuji

\title{
New radiological finding by magnetic resonance imaging examination of the brain in Coffin-Lowry syndrome
}

Received: July 23, 1997 / Accepted: October 24, 1997

\begin{abstract}
We used magnetic resonance imaging (MRI) to examine the brain of a typical Coffin-Lowry syndrome (CLS) patient. There were many small perivascular focal areas of hypointensity in the white matter on T1-weighted images, similar to those found in mucopolysaccharidosis or perivascular leukomalacia. However, these changes could not seen in another patient we examined. Both patients showed normal urinary mucopolysaccharide patterns with chromatographic analysis. The cause of the MRI result is not known, but it could have a heterogeneous origin, and this result could represent an important indication defining one type of CLS.
\end{abstract}

Key words Coffin-Lowry syndrome $\cdot$ Multiple perivascular space $\cdot$ Magnetic resonance imaging (MRI) - Mucopolysaccharidosis $\cdot$ Perivascular leukomalacia

\section{Introduction}

Coffin-Lowry syndrome (CLS) is a rare X-linked disorder which is characterized by facial dysmorphisms, tapering fingers, severe mental retardation, and progressive skeletal deformities (Young 1988). The radiographic signs, such as drumstick-shaped terminal phalangeal bones, a thick calvarium, narrow intervertebral spaces, and ligamentum flava calcification (Padley et al. 1990; Ishida et al. 1992; Miyazaki et al. 1990), are distinctive and help to provide confirmation of the diagnosis of this syndrome. Brain anomalies in this syndrome are uncommon but hydrocephalus and callosal

T. Kondoh $\cdot$ T. Matsumoto $\cdot$ Y. Tsuji $(\bowtie)$

Department of Pediatrics, Nagasaki University School of Medicine,

1-7-1 Sakamoto-machi, Nagasaki City, Nagasaki 852, Japan

Tel. +81-95-849-7298; Fax +81-95-849-7301

e-mail: pediatr@net.nagasaki-u.ac.jp

M. Ochi

Department of Radiology, Nagasaki University School of Medicine, Nagasaki, Japan

K. Sukegawa

Department of Pediatrics, Gifu University School of Medicine, Gifu, Japan dysgenesis have been reported (Ozden et al. 1994). We report a Japanese CLS patient who showed a distinctive brain abnormality when examined with magnetic resonance imaging (MRI).

\section{Case report}

The proband, a male, was the first infant born to a healthy 25 year-old G1P1 mother and a 26-year-old father. There were no family histories of consanguinity, congenital anomalies, or mental deficiency. The course of the pregnancy was uneventful except for a pelvic presentation. The child was born vaginally after 38 weeks' gestation, but with mild asphyxia and an Apgar score of 6 at $1 \mathrm{~min}$. His birth weight was $2850 \mathrm{~g}$. At 11 months of age, he was referred to us because of hypotonia and psychomotor retardation. He had a peculiar face including dolichocephaly, synophrys, anteverted nostrils, antimongoloid slant of palpebral fissures, right esotropia, a fish-like mouth, and large ears (Fig. 1a). Other signs were generalized hypotonicity, tapering fingers, a funnel chest, undescended testes, hyperhidrosis, mental retardation, and short stature (-2.8 SD). X-ray examination of his hand showed drumstick-shaped terminal phalanges (Fig. 1b). His bone age was accelerated to that of an 8-yearold, at a chronological age of 6 years.

From these clinical signs, he was diagnosed as a CoffinLowry syndrome patient. His karyotype was 46,XY. He is now a first-year student at a special educational school. His delayed development is not progressive. MRI of his brain demonstrated characteristic, multiple, well-defined, focal areas of hypointensity in the frontal white matter on parasagittal T1-weighted images (Fig. 2a,b). The areas showed isointensity to cerebrospinal fluid on T2-weighted images and on a proton density image (data not shown). These findings were thought to indicate dilated perivascular spaces caused by the deposition of metabolites such as mucopolysaccharides (Barkovich 1995) or perivascular leukomalacia (PVL). Dilatation of the lateral ventricles (Fig. 2) and hypoplasia of the splenium were also noted. 
Fig. 1 Morphological features (a) and an X-ray photograph of a hand (b) of the patient
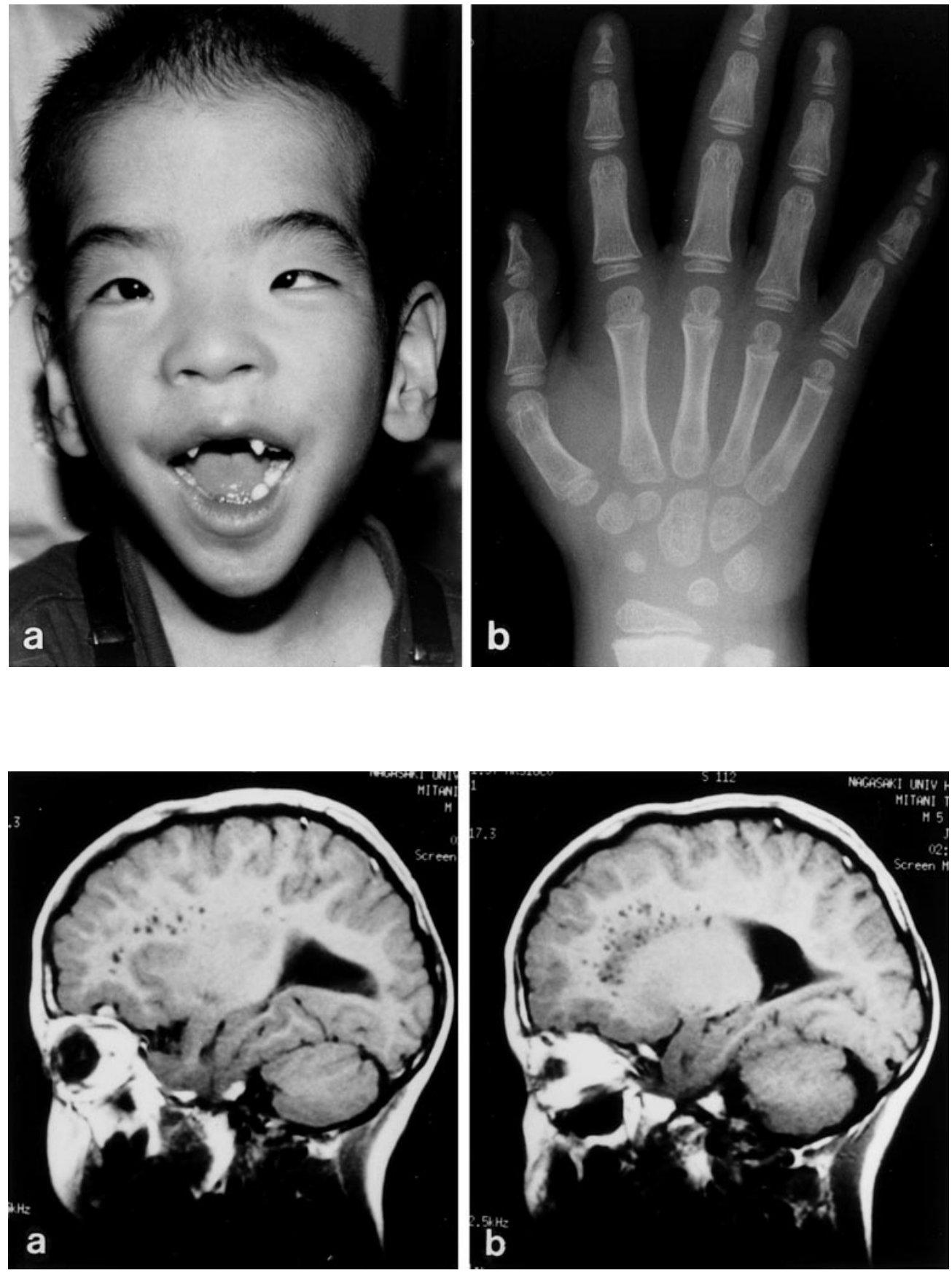

Fig. 2 T1-weighted magnetic resonance imaging photograph of the patient's brain at the different slices $(\mathrm{a}, \mathrm{b})$

The patient's urinary excretion level of mucopolysaccharides, including uronic acid, dermatan sulfate, heparan sulfate, chondroitin sulfate, and keratan sulfate, was within the normal range, as measured by densitometric analysis following electrophoresis (data not shown).

\section{Discussion}

Coffin-Lowry syndrome is a rare, X-linked, dysmorphological syndrome reported by Coffin et al. (1966).
The characteristic manifestations are a peculiar face, including hypertelorism, downward-slanted palpebral fissures, a short bulbous nose, anteverted nares, absent/ reduced crown of the permanent lower incisor, prominent square forehead, a thick everted lower lip, and other features such as severe mental retardation, short stature, pectus carinatum or excavatum, and tapering fingers (Young 1988).

The CLS gene was reported to be located on the short arm of X chromosome, Xp22.2-p22.1 (Biancalana et al. 1992, 1994). Some CLS patients have mutations of the Rsk-2 gene, which encodes a member of the growth-factor- 
regulated protein kinases, and is mapped within the CLS critical region (Trivier et al. 1996). However, CLS may be a heterogeneous disorder, because its clinical severity and complications are variable. There are some reports suggesting an association of CLS with metabolic disorders, such as abnormalities of proteodermatan sulfate (Vine et al. 1986), calcium pyrophosphate dehydrate crystal deposition (Ishida et al. 1992) and glycosaminoglycan (Miyazaki et al. 1990).

The MRI examination of the brain of our patient showed a new finding: multiple small T1 hypointensity regions. These closely resemble those found in mucopolysaccharidosis (MPS), in which hypointensity spots (the result of perivascular mucopolysaccharide deposition) are shown on T1-weighted images (Barkovich 1995). However, the facial features and other clinical signs of our patient were quiet different from those of MPS patients. His urinary excretion level of mucopolysaccharides was normal, suggesting that CLS is not caused by a mucopolysaccharide metabolic disorder. In addition, the patient showed mild dysgenesis of the corpus callosum which was compatible with CLS. We do not know the cause of the lesion in the patient which causes these hypointensity regions, or whether the hypointensity spots are related to the etiology of CLS or not. The MRI examinations were performed twice: in March 1991 and in January 1997. There seemed to be no difference in the number and distribution of the regions in his brain between the two examinations. This suggests that the changes might be caused by congenital malformations, not by progressive metabolic disorders. PVL is usually apparent in the anterior border zone between a ventriculopedal artery and a ventriculofugal artery of the brain in an immature baby with severe asphyxia (Banker and Larroche 1962). This patient was not an immature baby and was not affected by severe hypoxia. Moreover, the distribution of the affected region coincided with that of PVL.

MRI of the brain of another two typical CLS patients revealed mild dysgenesis of the corpus callosum, as in the present case, which is compatible with CLS. There were no hypointensity spots on the T1-weighted images. In the present case, the degree of mental retardation is more severe than those of the other two patients. There might be a relationship between mental retardation and the brain differences. Although our patient had typical morphological features of CLS, he showed a distinctive sign in the brain and an accelerated bone age. To our knowledge, these findings have not been reported previously. Our results suggest that CLS could be a heterogeneous disease.

\section{References}

Banker BD, Larroche JC (1962) Periventricular leukomalacia of infancy. Arch Neurol 7:386

Barkovich AJ (1995) Gray matter diseases and white matter diseases. In: Barkovich AJ (ed) Pediatric neuroimaging. Raven, New York, pp 58-99

Biancalana V, Briard ML, David A, Gilgenkrantz S, Kaplan J, Mathieu M, Piussan C, Poncin J, Schinzel A, Oudet C, Hanauer A (1992) Confirmation and refinement of the genetic localization of the Coffin-Lowry syndrome locus in Xp22.1-q22.2. Am J Hum Genet 50: 981-987

Biancalana V, Trivier E, Weber C, Weissenbach J, Rowe PSN, O'Riordan JLH, Partington MW, Heyberger S, Oudet C, Hanauer A (1994) Construction of a high-resolution linkage map for Xp22.1p22.2 and refinement of the genetic localization of the Coffin-Lowry syndrome gene. Genomics 22:205-213

Coffin GS, Siris E, Wegienka LC (1966) Mental retardation with osteocartilaginous anomalies. Am J Dis Child 112:205-213

Ishida Y, Oki T, Nogami H (1992) Coffin-Lowry syndrome associated with calcium pyrophosphate crystal deposition in the ligamenta flava. Clin Orthop 275:144-151

Miyazaki K, Yamanaka T, Ishida Y, Oohira A (1990) Calcified ligamenta flava in a patient with Coffin-Lowry syndrome: biochemical analysis of glycosaminoglycans. Jpn J Hum Genet 35:215-221

Ozden A, Dirik E, Emel A, Sevine N (1994) Callosal dysgenesis in a patient with Coffin-Lowry syndrome. Indian J Pediatr 61:101-103

Padley S, Hodgson SV, Sherwood T (1990) The radiology of CoffinLowry syndrome. Br J Radiol 63:72-75

Trivier E, de Cesare D, Jacquot S, Pannetier S, Zackai E, Young I, Mandel JL, Sassone-Corsi P, Hanauer A (1996) Mutations in the kinase Rsk-2 associated with Coffin-Lowry syndrome. Nature 384: $567-570$

Vine DT, Gold JT, Grant AD (1986) Etiology of the weakness in Coffin-Lowry syndrome. Am J Hum Genet 39:A85

Young ID (1988) The Coffin-Lowry syndrome. J Med Genet 26:344348 